\title{
A General Iterative Algorithm for Generalized Mixed Equilibrium Problems and Variational Inclusions Approach to Variational Inequalities
}

\author{
Thanyarat Jitpeera and Poom Kumam \\ Department of Mathematics, Faculty of Science, King Mongkut's University of \\ Technology Thonburi (KMUTT), Bangmod, Thrungkru, Bangkok 10140, Thailand \\ Correspondence should be addressed to Poom Kumam, poom.kum@kmutt.ac.th \\ Received 1 December 2010; Revised 28 January 2011; Accepted 17 February 2011 \\ Academic Editor: Vittorio Colao
}

Copyright (c) 2011 T. Jitpeera and P. Kumam. This is an open access article distributed under the Creative Commons Attribution License, which permits unrestricted use, distribution, and reproduction in any medium, provided the original work is properly cited.

\begin{abstract}
We introduce a new general iterative method for finding a common element of the set of solutions of fixed point for nonexpansive mappings, the set of solution of generalized mixed equilibrium problems, and the set of solutions of the variational inclusion for a $\beta$-inverse-strongly monotone mapping in a real Hilbert space. We prove that the sequence converges strongly to a common element of the above three sets under some mild conditions. Our results improve and extend the corresponding results of Marino and Xu (2006), Su et al. (2008), Klin-eam and Suantai (2009), Tan and Chang (2011), and some other authors.
\end{abstract}

\section{Introduction}

Let $C$ be a closed convex subset of a real Hilbert space $H$ with the inner product $\langle\cdot, \cdot\rangle$ and the norm $\|\cdot\|$. Let $F$ be a bifunction of $C \times C$ into $R$, where $R$ is the set of real numbers, $\Psi: C \rightarrow H$ a mapping, and $\varphi: C \rightarrow \mathcal{R}$ a real-valued function. The generalized mixed equilibrium problem is for finding $x \in C$ such that

$$
F(x, y)+\langle\Psi x, y-x\rangle+\varphi(y)-\varphi(x) \geq 0, \quad \forall y \in C
$$

The set of solutions of (1.1) is denoted by $\operatorname{GMEP}(F, \varphi, \Psi)$, that is,

$$
\operatorname{GMEP}(F, \varphi, \Psi)=\{x \in C: F(x, y)+\langle\Psi x, y-x\rangle+\varphi(y)-\varphi(x) \geq 0, \forall y \in C\}
$$


If $F \equiv 0$, the problem (1.1) is reduced into the mixed variational inequality of Browder type [1] for finding $x \in C$ such that

$$
\langle\Psi x, y-x\rangle+\varphi(y)-\varphi(x) \geq 0, \quad \forall y \in C
$$

The set of solutions of (1.3) is denoted by $\operatorname{MVI}(C, \varphi, \Psi)$.

If $\Psi \equiv 0$ and $\varphi \equiv 0$, the problem (1.1) is reduced into the equilibrium problem [2] for finding $x \in C$ such that

$$
F(x, y) \geq 0, \quad \forall y \in C
$$

The set of solutions of (1.4) is denoted by $\mathrm{EP}(F)$. This problem contains fixed point problems and includes as special cases numerous problems in physics, optimization, and economics. Some methods have been proposed to solve the equilibrium problem; see [3-5].

If $F \equiv 0$ and $\varphi \equiv 0$, the problem (1.1) is reduced into the Hartmann-Stampacchia variational inequality [6] for finding $x \in C$ such that

$$
\langle\Psi x, y-x\rangle \geq 0, \quad \forall y \in C
$$

The set of solutions of (1.5) is denoted by $\operatorname{VI}(C, \Psi)$. The variational inequality has been extensively studied in the literature [7].

If $F \equiv 0$ and $\Psi \equiv 0$, the problem (1.1) is reduced into the minimize problem for finding $x \in C$ such that

$$
\varphi(y)-\varphi(x) \geq 0, \quad \forall y \in C
$$

The set of solutions of (1.6) is denoted by $\operatorname{Arg} \min (\varphi)$.

Iterative methods for nonexpansive mappings have recently been applied to solve convex minimization problems. Convex minimization problems have a great impact and influence on the development of almost all branches of pure and applied sciences. A typical problem is to minimize a quadratic function over the set of the fixed points of a nonexpansive mapping on a real Hilbert space $H$ :

$$
\theta(x)=\frac{1}{2}\langle A x, x\rangle-\langle x, y\rangle, \quad \forall x \in F(S),
$$

where $A$ is a linear bounded operator, $F(S)$ is the fixed point set of a nonexpansive mapping $S$, and $y$ is a given point in $H$ [8].

Recall that a mapping $S: C \rightarrow C$ is said to be nonexpansive if

$$
\|S x-S y\| \leq\|x-y\|
$$


for all $x, y \in C$. If $C$ is bounded closed convex and $S$ is a nonexpansive mapping of $C$ into itself, then $F(S)$ is nonempty [9]. We denote weak convergence and strong convergence by notations $\rightarrow$ and $\rightarrow$, respectively. A mapping $A$ of $C$ into $H$ is called monotone if

$$
\langle A x-A y, x-y\rangle \geq 0
$$

for all $x, y \in C$. A mapping $A$ of $C$ into $H$ is called $\alpha$-inverse-strongly monotone if there exists a positive real number $\alpha$ such that

$$
\langle A x-A y, x-y\rangle \geq \alpha\|A x-A y\|^{2}
$$

for all $x, y \in C$. It is obvious that any $\alpha$-inverse-strongly monotone mapping $A$ is monotone and Lipschitz continuous mapping. A linear bounded operator $A$ is strongly positive if there exists a constant $\bar{\gamma}>0$ with the property

$$
\langle A x, x\rangle \geq \bar{\gamma}\|x\|^{2}
$$

for all $x \in H$. A self mapping $f: C \rightarrow C$ is a contractions on $C$ if there exists a constant $\alpha \in(0,1)$ such that

$$
\|f(x)-f(y)\| \leq \alpha\|x-y\|
$$

for all $x, y \in C$. We use $\prod_{C}$ to denote the collection of all contraction on $C$. Note that each $f \in \prod_{C}$ has a unique fixed point in $C$.

Let $B: H \rightarrow H$ be a single-valued nonlinear mapping and $M: H \rightarrow 2^{H}$ a set-valued mapping. The variational inclusion problem is to find $x \in H$ such that

$$
\theta \in B(x)+M(x)
$$

where $\theta$ is the zero vector in $H$. The set of solutions of problem (1.13) is denoted by $I(B, M)$. The variational inclusion has been extensively studied in the literature, see, for example, [1013] and the reference therein.

A set-valued mapping $M: H \rightarrow 2^{H}$ is called monotone if for all $x, y \in H, f \in M(x)$, and $g \in M(y)$ imply $\langle x-y, f-g\rangle \geq 0$. A monotone mapping $M$ is maximal if its graph $G(M):=\{(f, x) \in H \times H: f \in M(x)\}$ of $M$ is not properly contained in the graph of any other monotone mapping. It is known that a monotone mapping $M$ is maximal if and only if, for $(x, f) \in H \times H,\langle x-y, f-g\rangle \geq 0$ for all $(y, g) \in G(M)$ imply $f \in M(x)$.

Let $B$ be an inverse-strongly monotone mapping of $C$ into $H$, and let $N_{C} v$ be normal cone to $C$ at $v \in C$, that is, $N_{C} v=\{w \in H:\langle v-u, w\rangle \geq 0, \forall u \in C\}$, and define

$$
T v= \begin{cases}B v+N_{C} v, & \text { if } v \in C, \\ \emptyset, & \text { if } v \notin C .\end{cases}
$$

Then, $T$ is a maximal monotone and $\theta \in T v$ if and only if $v \in \operatorname{VI}(C, B)$ [14]. 
Let $M: H \rightarrow 2^{H}$ be a set-valued maximal monotone mapping; then the single-valued mapping $J_{M, \lambda}: H \rightarrow H$ defined by

$$
J_{M, \lambda}(x)=(I+\lambda M)^{-1}(x), \quad x \in H
$$

is called the resolvent operator associated with $M$, where $\lambda$ is any positive number and $I$ is the identity mapping. It is worth mentioning that the resolvent operator is nonexpansive, 1-inverse-strongly monotone and that a solution of problem (1.13) is a fixed point of the operator $J_{M, \lambda}(I-\lambda B)$ for all $\lambda>0[15]$.

In 2000, Moudafi [16] introduced the viscosity approximation method for nonexpansive mapping and proved that, if $H$ is a real Hilbert space, the sequence $\left\{x_{n}\right\}$ defined by the iterative method below, with the initial guess $x_{0} \in C$, is chosen arbitrarily,

$$
x_{n+1}=\alpha_{n} f\left(x_{n}\right)+\left(1-\alpha_{n}\right) S x_{n}, \quad n \geq 0,
$$

where $\left\{\alpha_{n}\right\} \subset(0,1)$ satisfies certain conditions and converges strongly to a fixed point of $S$ (say $\bar{x} \in C$ ) which is the unique solution of the following variational inequality:

$$
\langle(I-f) \bar{x}, x-\bar{x}\rangle \geq 0, \quad \forall x \in F(S)
$$

In 2006, Marino and $\mathrm{Xu}$ [8] introduced a general iterative method for nonexpansive mapping. They defined the sequence $\left\{x_{n}\right\}$ generated by the algorithm $x_{0} \in C$ :

$$
x_{n+1}=\alpha_{n} \gamma f\left(x_{n}\right)+\left(I-\alpha_{n} A\right) S x_{n}, \quad n \geq 0,
$$

where $\left\{\alpha_{n}\right\} \subset(0,1)$ and $A$ is a strongly positive linear bounded operator. They proved that, if $C=H$ and the sequence $\left\{\alpha_{n}\right\}$ satisfies appropriate conditions, then the sequence $\left\{x_{n}\right\}$ generated by (1.18) converges strongly to a fixed point of $S$ (say $\bar{x} \in H$ ) which is the unique solution of the following variational inequality:

$$
\langle(A-\gamma f) \bar{x}, x-\bar{x}\rangle \geq 0, \quad \forall x \in F(S)
$$

which is the optimality condition for the minimization problem

$$
\min _{x \in F(S) \cap E P(F)} \frac{1}{2}\langle A x, x\rangle-h(x),
$$

where $h$ is a potential function for $\gamma f$ (i.e., $h^{\prime}(x)=\gamma f(x)$ for $x \in H$ ). 
For finding a common element of the set of fixed points of nonexpansive mappings and the set of solution of the variational inequalities, let $P_{C}$ be the projection of $H$ onto $C$. In 2005, Iiduka and Takahashi [17] introduced following iterative process for $x_{0} \in C$ :

$$
x_{n+1}=\alpha_{n} u+\left(1-\alpha_{n}\right) S P_{C}\left(x_{n}-\lambda_{n} A x_{n}\right), \quad \forall n \geq 0,
$$

where $u \in C,\left\{\alpha_{n}\right\} \subset(0,1)$, and $\left\{\lambda_{n}\right\} \subset[a, b]$ for some $a, b$ with $0<a<b<2 \beta$. They proved that under certain appropriate conditions imposed on $\left\{\alpha_{n}\right\}$ and $\left\{\lambda_{n}\right\}$, the sequence $\left\{x_{n}\right\}$ generated by (1.21) converges strongly to a common element of the set of fixed points of nonexpansive mapping and the set of solutions of the variational inequality for an inversestrongly monotone mapping ( $\operatorname{say} \bar{x} \in C$ ) which solve some variational inequality

$$
\langle\bar{x}-u, x-\bar{x}\rangle \geq 0, \quad \forall x \in F(S) \cap \operatorname{VI}(C, A) .
$$

In 2008, Su et al. [18] introduced the following iterative scheme by the viscosity approximation method in a real Hilbert space: $x_{1}, u_{n} \in H$

$$
\begin{gathered}
F\left(u_{n}, y\right)+\frac{1}{r_{n}}\left\langle y-u_{n}, u_{n}-x_{n}\right\rangle \geq 0, \quad \forall y \in C, \\
x_{n+1}=\alpha_{n} f\left(x_{n}\right)+\left(1-\alpha_{n}\right) S P_{C}\left(u_{n}-\lambda_{n} A u_{n}\right),
\end{gathered}
$$

for all $n \in \mathbb{N}$, where $\left\{\alpha_{n}\right\} \subset[0,1)$ and $\left\{r_{n}\right\} \subset(0, \infty)$ satisfy some appropriate conditions. Furthermore, they proved that $\left\{x_{n}\right\}$ and $\left\{u_{n}\right\}$ converge strongly to the same point $z \in F(S) \cap$ $\mathrm{VI}(C, A) \cap \mathrm{EP}(F)$, where $z=P_{F(S) \cap \mathrm{VI}(C, A) \cap \mathrm{EP}(F)} f(z)$.

In 2011, Tan and Chang [12] introduced following iterative process for $\left\{T_{n}: C \rightarrow C\right\}$ which is a sequence of nonexpansive mappings. Let $\left\{x_{n}\right\}$ be the sequence defined by

$$
x_{n+1}=\alpha_{n} x_{n}+\left(1-\alpha_{n}\right)\left(S P_{C}\left(\left(1-t_{n}\right) J_{M, \lambda}(I-\lambda A) T_{\mu}(I-\mu B)\right) x_{n}\right), \quad \forall n \geq 0,
$$

where $\left\{\alpha_{n}\right\} \subset(0,1), \lambda \in(0,2 \alpha]$, and $\mu \in(0,2 \beta]$. The sequence $\left\{x_{n}\right\}$ defined by $(1.24)$ converges strongly to a common element of the set of fixed points of nonexpansive mapping, the set of solutions of the variational inequality, and the generalized equilibrium problem.

In this paper, we modify the iterative methods (1.18), (1.23), and (1.24) by proposing the following new general viscosity iterative method: $x_{0}, u_{n} \in C$,

$$
\begin{gathered}
F\left(u_{n}, y\right)+\varphi(y)-\varphi\left(u_{n}\right)+\left\langle\Psi x_{n}, y-u_{n}\right\rangle+\frac{1}{r_{n}}\left\langle y-u_{n}, u_{n}-x_{n}\right\rangle \geq 0, \quad \forall y \in C, \\
x_{n+1}=P_{C}\left[\alpha_{n} \gamma f\left(x_{n}\right)+\left(I-\alpha_{n} A\right) S J_{M, \lambda}\left(u_{n}-\lambda B u_{n}\right)\right]
\end{gathered}
$$

for all $n \in \mathbb{N}$, where $\left\{\alpha_{n}\right\} \subset(0,1),\left\{r_{n}\right\} \subset(0,2 \sigma)$, and $\lambda \in(0,2 \beta)$ satisfy some appropriate conditions. The purpose of this paper is to show that under some control conditions the sequence $\left\{x_{n}\right\}$ strongly converges to a common element of the set of fixed points of nonexpansive mapping, the solution of the generalized mixed equilibrium problems, and the set of solutions of the variational inclusion in a real Hilbert space. 


\section{Preliminaries}

Let $H$ be a real Hilbert space and $C$ a nonempty closed convex subset of $H$. Recall that the (nearest point) projection $P_{C}$ from $H$ onto $C$ assigns to each $x \in H$ the unique point in $P_{C} x \in C$ satisfying the property

$$
\left\|x-P_{C} x\right\|=\min _{y \in C}\|x-y\|
$$

The following characterizes the projection $P_{C}$. We recall some lemmas which will be needed in the rest of this paper.

Lemma 2.1. The function $u \in C$ is a solution of the variational inequality (1.5) if and only if $u \in C$ satisfies the relation $u=P_{C}(u-\lambda \Psi u)$ for all $\lambda>0$.

Lemma 2.2. For a given $z \in H, u \in C, u=P_{C} z \Leftrightarrow\langle u-z, v-u\rangle \geq 0$, for all $v \in C$.

It is well known that $P_{C}$ is a firmly nonexpansive mapping of $H$ onto $C$ and satisfies

$$
\left\|P_{C} x-P_{C} y\right\|^{2} \leq\left\langle P_{C} x-P_{C} y, x-y\right\rangle, \quad \forall x, y \in H
$$

Moreover, $P_{C} x$ is characterized by the following properties: $P_{C} x \in C$ and, for all $x \in H, y \in C$,

$$
\left\langle x-P_{C} x, y-P_{C} x\right\rangle \leq 0 .
$$

Lemma 2.3 (see [19]). Let $M: H \rightarrow 2^{H}$ be a maximal monotone mapping, and let $B: H \rightarrow H$ be a monotone and Lipshitz continuous mapping. Then the mapping $L=M+B: H \rightarrow 2^{H}$ is a maximal monotone mapping.

Lemma 2.4 (see [20]). Each Hilbert space $H$ satisfies Opial's condition, that is, for any sequence $\left\{x_{n}\right\} \subset H$ with $x_{n} \rightarrow x$, the inequality $\lim \inf _{n \rightarrow \infty}\left\|x_{n}-x\right\|<\lim _{\inf _{n \rightarrow \infty}}\left\|x_{n}-y\right\|$ holds for each $y \in H$ with $y \neq x$.

Lemma 2.5 (see [21]). Assume that $\left\{a_{n}\right\}$ is a sequence of nonnegative real numbers such that

$$
a_{n+1} \leq\left(1-\gamma_{n}\right) a_{n}+\delta_{n}, \quad \forall n \geq 0,
$$

where $\left\{\gamma_{n}\right\} \subset(0,1)$ and $\left\{\delta_{n}\right\}$ is a sequence in $R$ such that

(i) $\sum_{n=1}^{\infty} \gamma_{n}=\infty$,

(ii) $\lim \sup _{n \rightarrow \infty}\left(\delta_{n} / \gamma_{n}\right) \leq 0$ or $\sum_{n=1}^{\infty}\left|\delta_{n}\right|<\infty$.

Then $\lim _{n \rightarrow \infty} a_{n}=0$.

Lemma 2.6 (see [22]). Let $C$ be a closed convex subset of a real Hilbert space $H$, and let $T: C \rightarrow C$ be a nonexpansive mapping. Then $I-T$ is demiclosed at zero, that is,

$$
x_{n} \rightarrow x, \quad x_{n}-T x_{n} \longrightarrow 0
$$

implies $x=T x$. 
For solving the generalized mixed equilibrium problem, let us assume that the bifunction $F: C \times C \rightarrow R$, the nonlinear mapping $\Psi: C \rightarrow H$ is continuous monotone, and $\varphi: C \rightarrow \mathcal{R}$ satisfies the following conditions:

(A1) $F(x, x)=0$ for all $x \in C$;

(A2) $F$ is monotone, that is, $F(x, y)+F(y, x) \leq 0$ for any $x, y \in C$;

(A3) for each fixed $y \in C, x \mapsto F(x, y)$ is weakly upper semicontinuous;

(A4) for each fixed $x \in C, y \mapsto F(x, y)$ is convex and lower semicontinuous;

(B1) for each $x \in C$ and $r>0$, there exist a bounded subset $D_{x} \subseteq C$ and $y_{x} \in C$ such that, for any $z \in C \backslash D_{x}$,

$$
F\left(z, y_{x}\right)+\varphi\left(y_{x}\right)-\varphi(z)+\frac{1}{r}\left\langle y_{x}-z, z-x\right\rangle<0
$$

(B2) $C$ is a bounded set.

Lemma 2.7 (see [23]). Let $C$ be a nonempty closed convex subset of a real Hilbert space $H$. Let $F: C \times C \rightarrow R$ be a bifunction mapping satisfying (A1)-(A4), and let $\varphi: C \rightarrow R$ be convex and lower semicontinuous such that $C \cap \operatorname{dom} \varphi \neq \emptyset$. Assume that either (B1) or (B2) holds. For $r>0$ and $x \in H$, there exists $u \in C$ such that

$$
F(u, y)+\varphi(y)-\varphi(u)+\frac{1}{r}\langle y-u, u-x\rangle
$$

Define a mapping $K_{r}: H \rightarrow C$ as follows:

$$
K_{r}(x)=\left\{u \in C: F(u, y)+\varphi(y)-\varphi(u)+\frac{1}{r}\langle y-u, u-x\rangle \geq 0, \forall y \in C\right\}
$$

for all $x \in H$. Then, the following hold:

(i) $K_{r}$ is single valued;

(ii) $K_{r}$ is firmly nonexpansive, that is, for any $x, y \in H,\left\|K_{r} x-K_{r} y\right\|^{2} \leq\left\langle K_{r} x-K_{r} y, x-y\right\rangle$;

(iii) $F\left(K_{r}\right)=\operatorname{MEP}(F, \varphi)$;

(iv) $\operatorname{MEP}(F, \varphi)$ is closed and convex.

Lemma 2.8 (see [8]). Assume that $A$ is a strongly positive linear bounded operator on a Hilbert space $H$ with coefficient $\bar{\gamma}>0$ and $0<\rho \leq\|A\|^{-1}$; then $\|I-\rho A\| \leq 1-\rho \bar{\gamma}$.

\section{Strong Convergence Theorems}

In this section, we show a strong convergence theorem which solves the problem of finding a common element of $F(S), \operatorname{GMEP}(F, \varphi, \Psi)$, and $I(B, M)$ of an inverse-strongly monotone mappings in a Hilbert space. 
Theorem 3.1. Let $H$ be a real Hilbert space, $C$ a closed convex subset of $H, B, \Psi: C \rightarrow H$ be $\beta$, $\sigma$-inverse-strongly monotone mappings, respectively. Let $\varphi: C \rightarrow \mathcal{R}$ be a convex and lower semicontinuous function, $f: C \rightarrow C$ a contraction with coefficient $\alpha(0<\alpha<1), M: H \rightarrow 2^{H} a$ maximal monotone mapping, and $A$ a strongly positive linear bounded operator of $H$ into itself with coefficient $\bar{\gamma}>0$. Assume that $0<\gamma<\bar{\gamma} / \alpha$. Let $S$ be a nonexpansive mapping of $C$ into itself such that

$$
\Omega:=F(S) \cap \operatorname{GMEP}(F, \varphi, \Psi) \cap I(B, M) \neq \emptyset .
$$

Suppose that $\left\{x_{n}\right\}$ is a sequences generated by the following algorithm for $x_{0} \in C$ arbitrarily:

$$
\begin{gathered}
F\left(u_{n}, y\right)+\varphi(y)-\varphi\left(u_{n}\right)+\left\langle\Psi x_{n}, y-u_{n}\right\rangle+\frac{1}{r_{n}}\left\langle y-u_{n}, u_{n}-x_{n}\right\rangle \geq 0, \quad \forall y \in C, \\
x_{n+1}=P_{C}\left[\alpha_{n} \gamma f\left(x_{n}\right)+\left(I-\alpha_{n} A\right) S J_{M, \lambda}\left(u_{n}-\lambda B u_{n}\right)\right]
\end{gathered}
$$

for all $n=0,1,2, \ldots$, where

(C1) $\left\{\alpha_{n}\right\} \subset(0,1), \lim _{n \rightarrow 0} \alpha_{n}=0, \sum_{n=1}^{\infty} \alpha_{n}=\infty$, and $\sum_{n=1}^{\infty}\left|\alpha_{n+1}-\alpha_{n}\right|<\infty$,

(C2) $\left\{r_{n}\right\} \subset[c, d]$ with $c, d \in(0,2 \sigma)$ and $\sum_{n=1}^{\infty}\left|r_{n+1}-r_{n}\right|<\infty$,

(C3) $\lambda \in(0,2 \beta)$.

Then $\left\{x_{n}\right\}$ converges strongly to $q \in \Omega$, where $q=P_{\Omega}(\gamma f+I-A)(q)$ which solves the following variational inequality:

$$
\langle(\gamma f-A) q, p-q\rangle \leq 0, \quad \forall p \in \Omega
$$

which is the optimality condition for the minimization problem

$$
\min _{q \in \Omega} \frac{1}{2}\langle A q, q\rangle-h(q)
$$

where $h$ is a potential function for $\gamma f$ (i.e., $h^{\prime}(q)=\gamma f(q)$ for $q \in H$ ).

Proof. Due to condition (C1), we may assume without loss of generality, then, that $\alpha_{n} \in$ $\left(0,\|A\|^{-1}\right)$ for all $n$. By Lemma 2.8, we have that $\left\|I-\alpha_{n} A\right\| \leq 1-\alpha_{n} \bar{\gamma}$. Next, we will assume that $\|I-A\| \leq\|1-\bar{\gamma}\|$.

Next, we will divide the proof into six steps.

Step 1. We will show that $\left\{x_{n}\right\},\left\{u_{n}\right\}$ are bounded.

Since $B, \Psi$ are $\beta, \sigma$-inverse-strongly monotone mappings, we have that

$$
\begin{aligned}
\|(I-\lambda B) x-(I-\lambda B) y\|^{2} & =\|(x-y)-\lambda(B x-B y)\|^{2} \\
& =\|x-y\|^{2}-2 \lambda\langle x-y, B x-B y\rangle+\lambda^{2}\|B x-B y\|^{2} \\
& \leq\|x-y\|^{2}+\lambda(\lambda-2 \beta)\|B x-B y\|^{2} \\
& \leq\|x-y\|^{2} .
\end{aligned}
$$


In a similar way, we can obtain

$$
\left\|\left(I-r_{n} \Psi\right) x-\left(I-r_{n} \Psi\right) y\right\|^{2} \leq\|x-y\|^{2} .
$$

It is clear that if $0<\lambda<2 \beta, 0<r_{n}<2 \sigma$, then $I-\lambda B, I-r_{n} \Psi$ are all nonexpansive.

Put $y_{n}=J_{M, \lambda}\left(u_{n}-\lambda B u_{n}\right), n \geq 0$. It follows that

$$
\begin{aligned}
\left\|y_{n}-q\right\| & =\left\|J_{M, \lambda}\left(u_{n}-\lambda B u_{n}\right)-J_{M, \lambda}(q-\lambda B q)\right\| \\
& \leq\left\|u_{n}-q\right\| .
\end{aligned}
$$

By Lemma 2.7, we have that $u_{n}=K_{r_{n}}\left(x_{n}-r_{n} \Psi x_{n}\right)$ for all $n \geq 0$. Then, we have that

$$
\begin{aligned}
\left\|u_{n}-q\right\|^{2} & =\left\|K_{r_{n}}\left(x_{n}-r_{n} \Psi x_{n}\right)-K_{r_{n}}\left(q-r_{n} \Psi q\right)\right\|^{2} \\
& \leq\left\|\left(x_{n}-r_{n} \Psi x_{n}\right)-\left(q-r_{n} \Psi q\right)\right\|^{2} \\
& \leq\left\|x_{n}-q\right\|^{2}+r_{n}\left(r_{n}-2 \sigma\right)\left\|\Psi x_{n}-\Psi q\right\|^{2} \\
& =\left\|x_{n}-q\right\|^{2} .
\end{aligned}
$$

Hence, we have that

$$
\left\|y_{n}-q\right\| \leq\left\|x_{n}-q\right\|
$$

From (3.2), we deduce that

$$
\begin{aligned}
\left\|x_{n+1}-q\right\| & =\left\|P_{C}\left(\alpha_{n} \gamma f\left(x_{n}\right)+\left(I-\alpha_{n} A\right) S y_{n}\right)-P_{C}(q)\right\| \\
& \leq\left\|\alpha_{n}\left(\gamma f\left(x_{n}\right)-A q\right)+\left(I-\alpha_{n} A\right)\left(S y_{n}-q\right)\right\| \\
& \leq \alpha_{n}\left\|\gamma f\left(x_{n}\right)-A q\right\|+\left(1-\alpha_{n} \bar{\gamma}\right)\left\|y_{n}-q\right\| \\
& \leq \alpha_{n}\left\|\gamma f\left(x_{n}\right)-\gamma f(q)\right\|+\alpha_{n}\|\gamma f(q)-A q\|+\left(1-\alpha_{n} \bar{\gamma}\right)\left\|y_{n}-q\right\| \\
& \leq \alpha \gamma \alpha_{n}\left\|x_{n}-q\right\|+\alpha_{n}\|\gamma f(q)-A q\|+\left(1-\alpha_{n} \bar{\gamma}\right)\left\|x_{n}-q\right\| \\
& =\left(1-(\bar{\gamma}-\gamma \alpha) \alpha_{n}\right)\left\|x_{n}-q\right\|+\alpha_{n}\|\gamma f(q)-A q\| \\
& =\left(1-(\bar{\gamma}-\gamma \alpha) \alpha_{n}\right)\left\|x_{n}-q\right\|+(\bar{\gamma}-\gamma \alpha) \alpha_{n} \frac{\|\gamma f(q)-A q\|}{\bar{\gamma}-\gamma \alpha} \\
& \leq \max \left\{\left\|x_{n}-q\right\|, \frac{\|\gamma f(q)-A q\|}{\bar{\gamma}-\gamma \alpha}\right\} .
\end{aligned}
$$


It follows by induction that

$$
\left\|x_{n}-q\right\| \leq \max \left\{\left\|x_{0}-q\right\|, \frac{\|\gamma f(q)-A q\|}{\bar{\gamma}-\gamma \alpha}\right\}, \quad n \geq 0 .
$$

Therefore $\left\{x_{n}\right\}$ is bounded, so are $\left\{y_{n}\right\},\left\{S y_{n}\right\},\left\{B u_{n}\right\},\left\{f\left(x_{n}\right)\right\}$, and $\left\{A S y_{n}\right\}$.

Step 2. We claim that $\lim _{n \rightarrow \infty}\left\|x_{n+1}-x_{n}\right\|=0$. From (3.2), we have that

$$
\begin{aligned}
\left\|x_{n+1}-x_{n}\right\|= & \left\|P_{C}\left(\alpha_{n} \gamma f\left(x_{n}\right)+\left(I-\alpha_{n} A\right) S y_{n}\right)-P_{C}\left(\alpha_{n-1} \gamma f\left(x_{n-1}\right)+\left(I-\alpha_{n-1} A\right) S y_{n-1}\right)\right\| \\
\leq & \|\left(I-\alpha_{n} A\right)\left(S y_{n}-S y_{n-1}\right)-\left(\alpha_{n}-\alpha_{n-1}\right) A S y_{n-1} \\
& \quad+\gamma \alpha_{n}\left(f\left(x_{n}\right)-f\left(x_{n-1}\right)\right)+\gamma\left(\alpha_{n}-\alpha_{n-1}\right) f\left(x_{n-1}\right) \| \\
\leq & \left(1-\alpha_{n} \bar{\gamma}\right)\left\|y_{n}-y_{n-1}\right\|+\left|\alpha_{n}-\alpha_{n-1}\right|\left\|A S y_{n-1}\right\| \\
& +\gamma \alpha \alpha_{n}\left\|x_{n}-x_{n-1}\right\|+\gamma\left|\alpha_{n}-\alpha_{n-1}\right|\left\|f\left(x_{n-1}\right)\right\| .
\end{aligned}
$$

Since $I-\lambda B$ are nonexpansive, we also have that

$$
\begin{aligned}
\left\|y_{n}-y_{n-1}\right\| & =\left\|J_{M, \lambda}\left(u_{n}-\lambda B u_{n}\right)-J_{M, \lambda}\left(u_{n-1}-\lambda B u_{n-1}\right)\right\| \\
& \leq\left\|\left(u_{n}-\lambda B u_{n}\right)-\left(u_{n-1}-\lambda B u_{n-1}\right)\right\| \\
& \leq\left\|u_{n}-u_{n-1}\right\| .
\end{aligned}
$$

On the other hand, from $u_{n-1}=K_{r_{n-1}}\left(x_{n-1}-r_{n-1} \Psi x_{n-1}\right)$ and $u_{n}=K_{r_{n}}\left(x_{n}-r_{n} \Psi x_{n}\right)$, it follows that

$$
\begin{gathered}
F\left(u_{n-1}, y\right)+\left\langle\Psi x_{n-1}, y-u_{n-1}\right\rangle+\varphi(y)-\varphi\left(u_{n-1}\right)+\frac{1}{r_{n-1}}\left\langle y-u_{n-1}, u_{n-1}-x_{n-1}\right\rangle \geq 0, \quad \forall y \in C, \\
F\left(u_{n}, y\right)+\left\langle\Psi x_{n}, y-u_{n}\right\rangle+\varphi(y)-\varphi\left(u_{n}\right)+\frac{1}{r_{n}}\left\langle y-u_{n}, u_{n}-x_{n}\right\rangle \geq 0, \quad \forall y \in C .
\end{gathered}
$$

Substituting $y=u_{n}$ into (3.14) and $\mathrm{y}=u_{n-1}$ into (3.15), we get

$$
\begin{gathered}
F\left(u_{n-1}, u_{n}\right)+\left\langle\Psi x_{n-1}, u_{n}-u_{n-1}\right\rangle+\varphi\left(u_{n}\right)-\varphi\left(u_{n-1}\right)+\frac{1}{r_{n-1}}\left\langle u_{n}-u_{n-1}, u_{n-1}-x_{n-1}\right\rangle \geq 0, \\
F\left(u_{n}, u_{n-1}\right)+\left\langle\Psi x_{n}, u_{n-1}-u_{n}\right\rangle+\varphi\left(u_{n-1}\right)-\varphi\left(u_{n}\right)+\frac{1}{r_{n}}\left\langle u_{n-1}-u_{n}, u_{n}-x_{n}\right\rangle \geq 0 .
\end{gathered}
$$


International Journal of Mathematics and Mathematical Sciences

From (A2), we obtain

$$
\left\langle u_{n}-u_{n-1}, \Psi x_{n-1}-\Psi x_{n}+\frac{u_{n-1}-x_{n-1}}{r_{n-1}}-\frac{u_{n}-x_{n}}{r_{n}}\right\rangle \geq 0
$$

and then

$$
\left\langle u_{n}-u_{n-1}, r_{n-1}\left(\Psi x_{n-1}-\Psi x_{n}\right)+u_{n-1}-x_{n-1}-\frac{r_{n-1}}{r_{n}}\left(u_{n}-x_{n}\right)\right\rangle \geq 0
$$

So

$$
\left\langle u_{n}-u_{n-1}, r_{n-1} \Psi x_{n-1}-r_{n-1} \Psi x_{n}+u_{n-1}-u_{n}+u_{n}-x_{n-1}-\frac{r_{n-1}}{r_{n}}\left(u_{n}-x_{n}\right)\right\rangle \geq 0 .
$$

It follows that

$$
\begin{gathered}
\left\langle u_{n}-u_{n-1},\left(I-r_{n-1} \Psi\right) x_{n}-\left(I-r_{n-1} \Psi\right) x_{n-1}+u_{n-1}-u_{n}+u_{n}-x_{n}-\frac{r_{n-1}}{r_{n}}\left(u_{n}-x_{n}\right)\right\rangle \geq 0 \\
\left\langle u_{n}-u_{n-1}, u_{n-1}-u_{n}\right\rangle+\left\langle u_{n}-u_{n-1}, x_{n}-x_{n-1}+\left(1-\frac{r_{n-1}}{r_{n}}\right)\left(u_{n}-x_{n}\right)\right\rangle \geq 0 .
\end{gathered}
$$

Without loss of generality, let us assume that there exists a real number $c$ such that $r_{n-1}>c>0$, for all $n \in \mathbb{N}$. Then, we have that

$$
\begin{aligned}
\left\|u_{n}-u_{n-1}\right\|^{2} & \leq\left\langle u_{n}-u_{n-1}, x_{n}-x_{n-1}+\left(1-\frac{r_{n-1}}{r_{n}}\right)\left(u_{n}-x_{n}\right)\right\rangle \\
& \leq\left\|u_{n}-u_{n-1}\right\|\left\{\left\|x_{n}-x_{n-1}\right\|+\left|1-\frac{r_{n-1}}{r_{n}}\right|\left\|u_{n}-x_{n}\right\|\right\},
\end{aligned}
$$

and hence

$$
\begin{aligned}
\left\|u_{n}-u_{n-1}\right\| & \leq\left\|x_{n}-x_{n-1}\right\|+\frac{1}{r_{n}}\left|r_{n}-r_{n-1}\right|\left\|u_{n}-x_{n}\right\| \\
& \leq\left\|x_{n}-x_{n-1}\right\|+\frac{M_{1}}{c}\left|r_{n}-r_{n-1}\right|
\end{aligned}
$$


where $M_{1}=\sup \left\{\left\|u_{n}-x_{n}\right\|: n \in \mathbb{N}\right\}$. Substituting (3.22) into (3.13), we have that

$$
\left\|y_{n}-y_{n-1}\right\| \leq\left\|x_{n}-x_{n-1}\right\|+\frac{M_{1}}{c}\left|r_{n}-r_{n-1}\right|
$$

Substituting (3.23) into (3.12), we get

$$
\begin{aligned}
\left\|x_{n+1}-x_{n}\right\| \leq & \left(1-\alpha_{n} \bar{\gamma}\right)\left(\left\|x_{n}-x_{n-1}\right\|+\frac{M_{1}}{c}\left|r_{n}-r_{n-1}\right|\right)+\left|\alpha_{n}-\alpha_{n-1}\right|\left\|A S y_{n-1}\right\| \\
& +\gamma \alpha \alpha_{n}\left\|x_{n}-x_{n-1}\right\|+\gamma\left|\alpha_{n}-\alpha_{n-1}\right|\left\|f\left(x_{n-1}\right)\right\| \\
= & \left(1-\alpha_{n} \bar{\gamma}\right)\left\|x_{n}-x_{n-1}\right\|+\left(1-\alpha_{n} \bar{\gamma}\right) \frac{M_{1}}{c}\left|r_{n}-r_{n-1}\right|+\left|\alpha_{n}-\alpha_{n-1}\right|\left\|A S y_{n-1}\right\| \\
& +\gamma \alpha \alpha_{n}\left\|x_{n}-x_{n-1}\right\|+\gamma\left|\alpha_{n}-\alpha_{n-1}\right|\left\|f\left(x_{n-1}\right)\right\| \\
\leq & \left(1-(\bar{\gamma}-\gamma \alpha) \alpha_{n}\right)\left\|x_{n}-x_{n-1}\right\|+\frac{M_{1}}{c}\left|r_{n}-r_{n-1}\right|+\left|\alpha_{n}-\alpha_{n-1}\right|\left\|A S y_{n-1}\right\| \\
& +\gamma\left|\alpha_{n}-\alpha_{n-1}\right|\left\|f\left(x_{n-1}\right)\right\| \\
\leq & \left(1-(\bar{\gamma}-\gamma \alpha) \alpha_{n}\right)\left\|x_{n}-x_{n-1}\right\|+\frac{M_{1}}{c}\left|r_{n}-r_{n-1}\right|+M_{2}\left|\alpha_{n}-\alpha_{n-1}\right|,
\end{aligned}
$$

where $M_{2}=\sup \left\{\max \left\{\left\|A S y_{n-1}\right\|,\left\|f\left(x_{n-1}\right)\right\|: n \in \mathbb{N}\right\}\right\}$. By conditions (C1)-(C2) and Lemma 2.5 , we have that $\left\|x_{n+1}-x_{n}\right\| \rightarrow 0$ as $n \rightarrow \infty$. From (3.23), we also have that $\left\|y_{n+1}-y_{n}\right\| \rightarrow 0$ as $n \rightarrow \infty$.

Step 3. We show the following:

(i) $\lim _{n \rightarrow \infty}\left\|B u_{n}-B q\right\|=0$;

(ii) $\lim _{n \rightarrow \infty}\left\|\Psi x_{n}-\Psi q\right\|=0$.

For $q \in \Omega$ and $q=J_{M, \lambda}(q-\lambda B q)$, by (3.5) and (3.8), we get

$$
\begin{aligned}
\left\|y_{n}-q\right\|^{2} & =\left\|J_{M, \lambda}\left(u_{n}-\lambda B u_{n}\right)-J_{M, \lambda}(q-\lambda B q)\right\|^{2} \\
& \leq\left\|\left(u_{n}-\lambda B u_{n}\right)-(q-\lambda B q)\right\|^{2} \\
& \leq\left\|u_{n}-q\right\|^{2}+\lambda(\lambda-2 \beta)\left\|B u_{n}-B q\right\|^{2} \\
& \leq\left\|x_{n}-q\right\|^{2}+\lambda(\lambda-2 \beta)\left\|B u_{n}-B q\right\|^{2} .
\end{aligned}
$$


International Journal of Mathematics and Mathematical Sciences

It follows that

$$
\begin{aligned}
\left\|x_{n+1}-q\right\|^{2}= & \left\|P_{C}\left(\alpha_{n} \gamma f\left(x_{n}\right)+\left(I-\alpha_{n} A\right) S y_{n}\right)-P_{C}(q)\right\|^{2} \\
\leq & \left\|\alpha_{n}\left(\gamma f\left(x_{n}\right)-A q\right)+\left(I-\alpha_{n} A\right)\left(S y_{n}-q\right)\right\|^{2} \\
\leq & \left(\alpha_{n}\left\|\gamma f\left(x_{n}\right)-A q\right\|+\left(1-\alpha_{n} \bar{\gamma}\right)\left\|y_{n}-q\right\|\right)^{2} \\
\leq & \alpha_{n}\left\|\gamma f\left(x_{n}\right)-A q\right\|^{2}+\left(1-\alpha_{n} \bar{\gamma}\right)\left\|y_{n}-q\right\|^{2} \\
& +2 \alpha_{n}\left(1-\alpha_{n} \bar{\gamma}\right)\left\|\gamma f\left(x_{n}\right)-A q\right\|\left\|y_{n}-q\right\| \\
\leq & \alpha_{n}\left\|\gamma f\left(x_{n}\right)-A q\right\|^{2}+2 \alpha_{n}\left(1-\alpha_{n} \bar{\gamma}\right)\left\|\gamma f\left(x_{n}\right)-A q\right\|\left\|y_{n}-q\right\| \\
& +\left(1-\alpha_{n} \bar{\gamma}\right)\left(\left\|x_{n}-q\right\|^{2}+\lambda(\lambda-2 \beta)\left\|B u_{n}-B q\right\|^{2}\right) \\
\leq & \alpha_{n}\left\|\gamma f\left(x_{n}\right)-A q\right\|^{2}+2 \alpha_{n}\left(1-\alpha_{n} \bar{\gamma}\right)\left\|\gamma f\left(x_{n}\right)-A q\right\|\left\|y_{n}-q\right\| \\
& +\left\|x_{n}-q\right\|^{2}+\left(1-\alpha_{n} \bar{\gamma}\right) \lambda(\lambda-2 \beta)\left\|B u_{n}-B q\right\|^{2} .
\end{aligned}
$$

So, we obtain

$$
\begin{aligned}
& \left(1-\alpha_{n} \bar{\gamma}\right) \lambda(2 \beta-\lambda)\left\|B u_{n}-B q\right\|^{2} \\
& \quad \leq \alpha_{n}\left\|\gamma f\left(x_{n}\right)-A q\right\|^{2}+\left\|x_{n}-x_{n+1}\right\|\left(\left\|x_{n}-q\right\|+\left\|x_{n+1}-q\right\|\right)+\epsilon_{n}
\end{aligned}
$$

where $\epsilon_{n}=2 \alpha_{n}\left(1-\alpha_{n} \bar{\gamma}\right)\left\|\gamma f\left(x_{n}\right)-A q\right\|\left\|y_{n}-q\right\|$. By conditions (C1) and (C3) and $\lim _{n \rightarrow \infty} \| x_{n+1}-$ $x_{n} \|=0$, we obtain that $\left\|B u_{n}-B q\right\| \rightarrow 0$ as $n \rightarrow \infty$.

Substituting (3.8) into (3.25), we get

$$
\begin{aligned}
\left\|y_{n}-q\right\|^{2} & \leq\left\|u_{n}-q\right\|^{2}+\lambda(\lambda-2 \beta)\left\|B u_{n}-B q\right\|^{2} \\
& \leq\left(\left\|x_{n}-q\right\|^{2}+r_{n}\left(r_{n}-2 \sigma\right)\left\|\Psi x_{n}-\Psi q\right\|^{2}\right)+\lambda(\lambda-2 \beta)\left\|B u_{n}-B q\right\|^{2} .
\end{aligned}
$$

From (3.26), we have that

$$
\begin{aligned}
\left\|x_{n+1}-q\right\|^{2} \leq & \alpha_{n}\left\|\gamma f\left(x_{n}\right)-A q\right\|^{2}+2 \alpha_{n}\left(1-\alpha_{n} \bar{\gamma}\right)\left\|\gamma f\left(x_{n}\right)-A q\right\|\left\|y_{n}-q\right\| \\
& +\left(1-\alpha_{n} \bar{\gamma}\right)\left(\left\|x_{n}-q\right\|^{2}+r_{n}\left(r_{n}-2 \sigma\right)\left\|\Psi x_{n}-\Psi q\right\|^{2}+\lambda(\lambda-2 \beta)\left\|B u_{n}-B q\right\|^{2}\right) \\
\leq & \alpha_{n}\left\|\gamma f\left(x_{n}\right)-A q\right\|^{2}+2 \alpha_{n}\left(1-\alpha_{n} \bar{\gamma}\right)\left\|\gamma f\left(x_{n}\right)-A q\right\|\left\|y_{n}-q\right\|+\left\|x_{n}-q\right\|^{2} \\
& +\left(1-\alpha_{n} \bar{\gamma}\right) r_{n}\left(r_{n}-2 \sigma\right)\left\|\Psi x_{n}-\Psi q\right\|^{2}+\left(1-\alpha_{n} \bar{\gamma}\right) \lambda(\lambda-2 \beta)\left\|B u_{n}-B q\right\|^{2} .
\end{aligned}
$$


So, we also have that

$$
\begin{aligned}
\left(1-\alpha_{n} \bar{\gamma}\right) r_{n}\left(2 \sigma-r_{n}\right)\left\|\Psi x_{n}-\Psi q\right\|^{2} \\
\leq \alpha_{n}\left\|\gamma f\left(x_{n}\right)-A q\right\|^{2}+\left\|x_{n}-x_{n+1}\right\|\left(\left\|x_{n}-q\right\|+\left\|x_{n+1}-q\right\|\right) \\
\quad+\epsilon_{n}+\left(1-\alpha_{n} \bar{\gamma}\right) \lambda(\lambda-2 \beta)\left\|B u_{n}-B q\right\|^{2}
\end{aligned}
$$

where $\epsilon_{n}=2 \alpha_{n}\left(1-\alpha_{n} \bar{\gamma}\right)\left\|\gamma f\left(x_{n}\right)-A q\right\|\left\|y_{n}-q\right\|$. By conditions (C1)-(C3), $\lim _{n \rightarrow \infty}\left\|x_{n+1}-x_{n}\right\|=0$ and $\lim _{n \rightarrow \infty}\left\|B u_{n}-B q\right\|=0$, we obtain that $\left\|\Psi x_{n}-\Psi q\right\| \rightarrow 0$ as $n \rightarrow \infty$.

Step 4. We show the following:

(i) $\lim _{n \rightarrow \infty}\left\|x_{n}-u_{n}\right\|=0$;

(ii) $\lim _{n \rightarrow \infty}\left\|u_{n}-y_{n}\right\|=0$;

(iii) $\lim _{n \rightarrow \infty}\left\|y_{n}-S y_{n}\right\|=0$.

Since $K_{r_{n}}$ is firmly nonexpansive and by (2.2), we observe that

$$
\begin{aligned}
\left\|u_{n}-q\right\|^{2}= & \left\|K_{r_{n}}\left(x_{n}-r_{n} \Psi x_{n}\right)-K_{r_{n}}\left(q-r_{n} \Psi q\right)\right\|^{2} \\
\leq & \left\langle\left(x_{n}-r_{n} \Psi x_{n}\right)-\left(q-r_{n} \Psi q\right), u_{n}-q\right\rangle \\
= & \frac{1}{2}\left(\left\|\left(x_{n}-r_{n} \Psi x_{n}\right)-\left(q-r_{n} \Psi q\right)\right\|^{2}+\left\|u_{n}-q\right\|^{2}\right. \\
& \left.\quad-\left\|\left(x_{n}-r_{n} \Psi x_{n}\right)-\left(q-r_{n} \Psi q\right)-\left(u_{n}-q\right)\right\|^{2}\right) \\
\leq & \frac{1}{2}\left(\left\|x_{n}-q\right\|^{2}+\left\|u_{n}-q\right\|^{2}-\left\|\left(x_{n}-u_{n}\right)-r_{n}\left(\Psi x_{n}-\Psi q\right)\right\|^{2}\right) \\
= & \frac{1}{2}\left(\left\|x_{n}-q\right\|^{2}+\left\|u_{n}-q\right\|^{2}-\left\|x_{n}-u_{n}\right\|^{2}\right. \\
& \left.+2 r_{n}\left\langle\Psi x_{n}-\Psi q, x_{n}-u_{n}\right\rangle-r_{n}^{2}\left\|\Psi x_{n}-\Psi q\right\|^{2}\right) .
\end{aligned}
$$

Hence, we have that

$$
\left\|u_{n}-q\right\|^{2} \leq\left\|x_{n}-q\right\|^{2}-\left\|x_{n}-u_{n}\right\|^{2}+2 r_{n}\left\|\Psi x_{n}-\Psi q\right\|\left\|x_{n}-u_{n}\right\|
$$


International Journal of Mathematics and Mathematical Sciences

Since $J_{M, \lambda}$ is 1-inverse-strongly monotone and by (2.2), we compute

$$
\begin{aligned}
\left\|y_{n}-q\right\|^{2}= & \left\|J_{M, \lambda}\left(u_{n}-\lambda B u_{n}\right)-J_{M, \lambda}(q-\lambda B q)\right\|^{2} \\
\leq & \left\langle\left(u_{n}-\lambda B u_{n}\right)-(q-\lambda B q), y_{n}-q\right\rangle \\
= & \frac{1}{2}\left(\left\|\left(u_{n}-\lambda B u_{n}\right)-(q-\lambda B q)\right\|^{2}+\left\|y_{n}-q\right\|^{2}\right. \\
& \left.\quad-\left\|\left(u_{n}-\lambda B u_{n}\right)-(q-\lambda B q)-\left(y_{n}-q\right)\right\|^{2}\right) \\
\leq & \frac{1}{2}\left(\left\|u_{n}-q\right\|^{2}+\left\|y_{n}-q\right\|^{2}-\left\|\left(u_{n}-y_{n}\right)-\lambda\left(B u_{n}-B q\right)\right\|^{2}\right) \\
= & \frac{1}{2}\left(\left\|u_{n}-q\right\|^{2}+\left\|y_{n}-q\right\|^{2}-\left\|u_{n}-y_{n}\right\|^{2}\right. \\
& \left.+2 \lambda\left\langle u_{n}-y_{n}, B u_{n}-B q\right\rangle-\lambda^{2}\left\|B u_{n}-B q\right\|^{2}\right),
\end{aligned}
$$

which implies that

$$
\left\|y_{n}-q\right\|^{2} \leq\left\|u_{n}-q\right\|^{2}-\left\|u_{n}-y_{n}\right\|^{2}+2 \lambda\left\|u_{n}-y_{n}\right\|\left\|B u_{n}-B q\right\| .
$$

Substituting (3.32) into (3.34), we have that

$$
\begin{aligned}
\left\|y_{n}-q\right\|^{2} \leq & \left(\left\|x_{n}-q\right\|^{2}-\left\|x_{n}-u_{n}\right\|^{2}+2 r_{n}\left\|\Psi x_{n}-\Psi q\right\|\left\|x_{n}-u_{n}\right\|\right) \\
& -\left\|u_{n}-y_{n}\right\|^{2}+2 \lambda\left\|u_{n}-y_{n}\right\|\left\|B u_{n}-B q\right\| .
\end{aligned}
$$

Substituting (3.35) into (3.26), we get

$$
\begin{gathered}
\left\|x_{n+1}-q\right\|^{2} \leq \alpha_{n}\left\|\gamma f\left(x_{n}\right)-A q\right\|^{2}+\left\|y_{n}-q\right\|^{2}+2 \alpha_{n}\left(1-\alpha_{n} \bar{\gamma}\right)\left\|\gamma f\left(x_{n}\right)-A q\right\|\left\|y_{n}-q\right\| \\
\leq \alpha_{n}\left\|\gamma f\left(x_{n}\right)-A q\right\|^{2}+\left(\left\|x_{n}-q\right\|^{2}-\left\|x_{n}-u_{n}\right\|^{2}+2 r_{n}\left\|\Psi x_{n}-\Psi q\right\|\left\|x_{n}-u_{n}\right\|\right. \\
\left.-\left\|u_{n}-y_{n}\right\|^{2}+2 \lambda_{n}\left\|u_{n}-y_{n}\right\|\left\|B u_{n}-B q\right\|\right) \\
+2 \alpha_{n}\left(1-\alpha_{n} \bar{\gamma}\right)\left\|\gamma f\left(x_{n}\right)-A q\right\|\left\|y_{n}-q\right\| .
\end{gathered}
$$


Then, we derive

$$
\begin{aligned}
\left\|x_{n}-u_{n}\right\|^{2}+\left\|u_{n}-y_{n}\right\|^{2} \leq & \alpha_{n}\left\|\gamma f\left(x_{n}\right)-A q\right\|^{2}+\left\|x_{n}-q\right\|^{2}-\left\|x_{n+1}-q\right\|^{2} \\
& +2 r_{n}\left\|\Psi x_{n}-\Psi q\right\|\left\|x_{n}-u_{n}\right\|+2 \lambda\left\|u_{n}-y_{n}\right\|\left\|B u_{n}-B q\right\| \\
& +2 \alpha_{n}\left(1-\alpha_{n} \bar{\gamma}\right)\left\|\gamma f\left(x_{n}\right)-A q\right\|\left\|y_{n}-q\right\| \\
= & \alpha_{n}\left\|\gamma f\left(x_{n}\right)-A q\right\|^{2}+\left\|x_{n}-x_{n+1}\right\|\left(\left\|x_{n}-q\right\|+\left\|x_{n+1}-q\right\|\right) \\
& +2 r_{n}\left\|\Psi x_{n}-\Psi q\right\|\left\|x_{n}-u_{n}\right\|+2 \lambda\left\|u_{n}-y_{n}\right\|\left\|B u_{n}-B q\right\| \\
& +2 \alpha_{n}\left(1-\alpha_{n} \bar{\gamma}\right)\left\|\gamma f\left(x_{n}\right)-A q\right\|\left\|y_{n}-q\right\| .
\end{aligned}
$$

By condition (C1), $\lim _{n \rightarrow \infty}\left\|x_{n}-x_{n+1}\right\|=0, \lim _{n \rightarrow \infty}\left\|\Psi x_{n}-\Psi q\right\|=0$, and $\lim _{n \rightarrow \infty}\left\|B u_{n}-B q\right\|=0$. So, we have that $\left\|x_{n}-u_{n}\right\| \rightarrow 0,\left\|u_{n}-y_{n}\right\| \rightarrow 0$ as $n \rightarrow \infty$. It follows that

$$
\left\|x_{n}-y_{n}\right\| \leq\left\|x_{n}-u_{n}\right\|+\left\|u_{n}-y_{n}\right\| \longrightarrow 0, \quad \text { as } n \longrightarrow \infty
$$

From (3.2), we have that

$$
\begin{aligned}
\left\|x_{n}-S y_{n}\right\| & \leq\left\|x_{n}-S y_{n-1}\right\|+\left\|S y_{n-1}-S y_{n}\right\| \\
& \leq\left\|P_{C}\left(\alpha_{n-1} \gamma f\left(x_{n-1}\right)+\left(I-\alpha_{n-1} A\right) S y_{n-1}\right)-P_{C}\left(S y_{n-1}\right)\right\|+\left\|y_{n-1}-y_{n}\right\| \\
& \leq \alpha_{n-1}\left\|\gamma f x_{n-1}-A S y_{n-1}\right\|+\left\|y_{n-1}-y_{n}\right\| .
\end{aligned}
$$

By condition (C1) and $\lim _{n \rightarrow \infty}\left\|y_{n-1}-y_{n}\right\|=0$, we obtain that $\left\|x_{n}-S y_{n}\right\| \rightarrow 0$ as $n \rightarrow \infty$. Next, we observe that

$$
\begin{aligned}
\left\|x_{n+1}-S y_{n}\right\| & =\left\|P_{C}\left(\alpha_{n} \gamma f\left(x_{n}\right)+\left(I-\alpha_{n} A\right) S y_{n}\right)-P_{C}\left(S y_{n}\right)\right\| \\
& \leq\left\|\alpha_{n} \gamma f\left(x_{n}\right)+\left(I-\alpha_{n} A\right) S y_{n}-S y_{n}\right\| \\
& =\alpha_{n}\left\|\gamma f\left(x_{n}\right)-A S y_{n}\right\| .
\end{aligned}
$$

Since $\left\{A S y_{n}\right\}$ is bounded and by condition (C1), we have that $\left\|x_{n+1}-S y_{n}\right\| \rightarrow 0$ as $n \rightarrow \infty$, and

$$
\left\|x_{n}-S y_{n}\right\| \leq\left\|x_{n}-x_{n+1}\right\|+\left\|x_{n+1}-S y_{n}\right\| \text {. }
$$

Since $\lim _{n \rightarrow \infty}\left\|x_{n}-x_{n+1}\right\|=0$ and $\lim _{n \rightarrow \infty}\left\|x_{n+1}-S y_{n}\right\|=0$, it implies that $\left\|x_{n}-S y_{n}\right\| \rightarrow 0$ as $n \rightarrow \infty$. Hence, we have that

$$
\begin{aligned}
\left\|x_{n}-S x_{n}\right\| & \leq\left\|x_{n}-S y_{n}\right\|+\left\|S y_{n}-S x_{n}\right\| \\
& \leq\left\|x_{n}-S y_{n}\right\|+\left\|y_{n}-x_{n}\right\| .
\end{aligned}
$$


By (3.38) and $\lim _{n \rightarrow \infty}\left\|x_{n}-S y_{n}\right\|=0$, we obtain $\left\|x_{n}-S x_{n}\right\| \rightarrow 0$ as $n \rightarrow \infty$. Moreover, we also have that

$$
\left\|y_{n}-S y_{n}\right\| \leq\left\|y_{n}-x_{n}\right\|+\left\|x_{n}-S y_{n}\right\|
$$

By (3.38) and $\lim _{n \rightarrow \infty}\left\|x_{n}-S y_{n}\right\|=0$, we obtain $\left\|y_{n}-S y_{n}\right\| \rightarrow 0$ as $n \rightarrow \infty$.

Step 5. We show that $q \in \Omega:=F(S) \cap \operatorname{GMEP}(F, \varphi, \Psi) \cap I(B, M)$ and $\limsup _{n \rightarrow \infty}\langle(\gamma f-$ A) $\left.q, S y_{n}-q\right\rangle \leq 0$. It is easy to see that $P_{\Omega}(\gamma f+(I-A))$ is a contraction of $H$ into itself. Indeed, since $0<\gamma<\bar{\gamma} / \alpha$, we have that

$$
\begin{aligned}
\left\|P_{\Omega}(\gamma f+(I-A)) x-P_{\Omega}(\gamma f+(I-A)) y\right\| & \leq\|\gamma f+(I-A) x-\gamma f-(I-A) y\| \\
& \leq \gamma\|f(x)-f(y)\|+\|I-A\|\|x-y\| \\
& \leq \gamma \alpha\|x-y\|+(1-\bar{\gamma})\|x-y\| \\
& \leq(1-\bar{\gamma}+\gamma \alpha)\|x-y\| .
\end{aligned}
$$

Hence $H$ is complete, and there exists a unique fixed point $q \in H$ such that $q=P_{\Omega}(\gamma f+(I-$ A))(q). By Lemma 2.2, we obtain that $\langle(\gamma f-A) q, w-q\rangle \leq 0$ for all $w \in \Omega$.

Next, we show that $\limsup _{n \rightarrow \infty}\left\langle(\gamma f-A) q, S y_{n}-q\right\rangle \leq 0$, where $q=P_{\Omega}(\gamma f+I-A)(q)$ is the unique solution of the variational inequality $\langle(\gamma f-A) q, p-q\rangle \geq 0$, for all $p \in \Omega$. We can choose a subsequence $\left\{y_{n_{i}}\right\}$ of $\left\{y_{n}\right\}$ such that

$$
\limsup _{n \rightarrow \infty}\left\langle(\gamma f-A) q, S y_{\mathrm{n}}-q\right\rangle=\lim _{i \rightarrow \infty}\left\langle(\gamma f-A) q, S y_{n_{i}}-q\right\rangle
$$

As $\left\{y_{n_{i}}\right\}$ is bounded, there exists a subsequence $\left\{y_{n_{i j}}\right\}$ of $\left\{y_{n_{i}}\right\}$ which converges weakly to $w$. We may assume without loss of generality that $y_{n_{i}} \rightarrow w$.

We claim that $w \in \Omega$. Since $\left\|y_{n}-S y_{n}\right\| \rightarrow 0,\left\|x_{n}-S x_{n}\right\| \rightarrow 0$, and $\left\|x_{n}-y_{n}\right\| \rightarrow 0$ and by Lemma 2.6, we have that $w \in F(S)$.

Next, we show that $w \in \operatorname{GMEP}(F, \varphi, \Psi)$. Since $u_{n}=K_{r_{n}}\left(x_{n}-r_{n} \Psi x_{n}\right)$, we know that

$$
F\left(u_{n}, y\right)+\varphi(y)-\varphi\left(u_{n}\right)+\left\langle\Psi x_{n}, y-u_{n}\right\rangle+\frac{1}{r_{n}}\left\langle y-u_{n}, u_{n}-x_{n}\right\rangle \geq 0, \quad \forall y \in C
$$

It follows by (A2) that

$$
\varphi(y)-\varphi\left(u_{n}\right)+\left\langle\Psi x_{n}, y-u_{n}\right\rangle+\frac{1}{r_{n}}\left\langle y-u_{n}, u_{n}-x_{n}\right\rangle \geq F\left(y, u_{n}\right), \quad \forall y \in C
$$

Hence,

$$
\varphi(y)-\varphi\left(u_{n_{i}}\right)+\left\langle\Psi x_{n_{i}}, y-u_{n_{i}}\right\rangle+\frac{1}{r_{n_{i}}}\left\langle y-u_{n_{i}}, u_{n_{i}}-x_{n_{i}}\right\rangle \geq F\left(y, u_{n_{i}}\right), \quad \forall y \in C .
$$


For $t \in(0,1]$ and $y \in H$, let $y_{t}=t y+(1-t) w$. From (3.48), we have that

$$
\begin{aligned}
\left\langle y_{t}-u_{n_{i}}, \Psi y_{t}\right\rangle \geq & \left\langle y_{t}-u_{n_{i}}, \Psi y_{t}\right\rangle-\varphi\left(y_{t}\right)+\varphi\left(u_{n_{i}}\right)-\left\langle\Psi x_{n_{i}}, y_{t}-u_{n_{i}}\right\rangle \\
& -\frac{1}{r_{n_{i}}}\left\langle y_{t}-u_{n_{i}}, u_{n_{i}}-x_{n_{i}}\right\rangle+F\left(y_{t}, u_{n_{i}}\right) \\
= & \left\langle y_{t}-u_{n_{i}}, \Psi y_{t}-\Psi u_{n_{i}}\right\rangle+\left\langle y_{t}-u_{n_{i}}, \Psi u_{n_{i}}-\Psi x_{n_{i}}\right\rangle-\varphi\left(y_{t}\right)+\varphi\left(u_{n_{i}}\right) \\
& -\frac{1}{\mathrm{r}_{n_{i}}}\left\langle y_{t}-u_{n_{i}}, u_{n_{i}}-x_{n_{i}}\right\rangle+F\left(y_{t}, u_{n_{i}}\right) .
\end{aligned}
$$

From $\left\|u_{n_{i}}-x_{n_{i}}\right\| \rightarrow 0$, we have that $\left\|\Psi u_{n_{i}}-\Psi x_{n_{i}}\right\| \rightarrow 0$. Further, from (A4) and the weakly lower semicontinuity of $\varphi,\left(u_{n_{i}}-x_{n_{i}}\right) / r_{n_{i}} \rightarrow 0$ and $u_{n_{i}} \rightarrow w$, we have that

$$
\left\langle y_{t}-w, \Psi y_{t}\right\rangle \geq-\varphi\left(y_{t}\right)+\varphi(w)+F\left(y_{t}, w\right)
$$

From (A1), (A4), and (3.50), we have that

$$
\begin{aligned}
0 & =F\left(y_{t}, y_{t}\right)-\varphi\left(y_{t}\right)+\varphi\left(y_{t}\right) \\
& \leq t F\left(y_{t}, y\right)+(1-t) F\left(y_{t}, w\right)+t \varphi(y)+(1-t) \varphi(w)-\varphi\left(y_{t}\right) \\
& =t\left[F\left(y_{t}, y\right)+\varphi(y)-\varphi\left(y_{t}\right)\right]+(1-t)\left[F\left(y_{t}, w\right)+\varphi(w)-\varphi\left(y_{t}\right)\right] \\
& \leq t\left[F\left(y_{t}, y\right)+\varphi(y)-\varphi\left(y_{t}\right)\right]+(1-t)\left\langle y_{t}-w, \Psi y_{t}\right\rangle \\
& =t\left[F\left(y_{t}, y\right)+\varphi(y)-\varphi\left(y_{t}\right)\right]+(1-t) t\left\langle y-w, \Psi y_{t}\right\rangle,
\end{aligned}
$$

and hence

$$
0 \leq F\left(y_{t}, y\right)+\varphi(y)-\varphi\left(y_{t}\right)+(1-t)\left\langle y-w, \Psi y_{t}\right\rangle
$$

Letting $t \rightarrow 0$, we have, for each $y \in C$, that

$$
F(w, y)+\varphi(y)-\varphi(w)+\langle y-w, \Psi w\rangle \geq 0
$$

This implies that $w \in \operatorname{GMEP}(F, \varphi, \Psi)$.

Lastly, we show that $w \in I(B, M)$. In fact, since $B$ is a $\beta$-inverse-strongly monotone, $B$ is monotone and Lipschitz continuous mapping. It follows from Lemma 2.3 that $M+B$ is a maximal monotone. Let $(v, g) \in G(M+B)$, since $g-B v \in M(v)$. Again since $y_{n_{i}}=J_{M, \lambda}\left(u_{n_{i}}-\right.$ $\left.\lambda B u_{n_{i}}\right)$, we have that $u_{n_{i}}-\lambda B u_{n_{i}} \in(I+\lambda M)\left(y_{n_{i}}\right)$, that is, $(1 / \lambda)\left(u_{n_{i}}-y_{n_{i}}-\lambda B u_{n_{i}}\right) \in M\left(y_{n_{i}}\right)$. By virtue of the maximal monotonicity of $M+B$, we have that

$$
\left\langle v-y_{n_{i}}, g-B v-\frac{1}{\lambda}\left(u_{n_{i}}-y_{n_{i}}-\lambda B u_{n_{i}}\right)\right\rangle \geq 0,
$$


and hence

$$
\begin{aligned}
\left\langle v-y_{n_{i}}, g\right\rangle \geq & \left\langle v-y_{n_{i}}, B v+\frac{1}{\lambda}\left(u_{n_{i}}-y_{n_{i}}-\lambda B u_{n_{i}}\right)\right\rangle \\
= & \left\langle v-y_{n_{i}}, B v-B y_{n_{i}}\right\rangle+\left\langle v-y_{n_{i}}, B y_{n_{i}}-B u_{n_{i}}\right\rangle \\
& +\left\langle v-y_{n_{i}}, \frac{1}{\lambda}\left(u_{n_{i}}-y_{n_{i}}\right)\right\rangle .
\end{aligned}
$$

It follows from $\lim _{n \rightarrow \infty}\left\|u_{n}-y_{n}\right\|=0, \lim _{n \rightarrow \infty}\left\|B u_{n}-B y_{n}\right\|=0$, and $y_{n_{i}} \rightarrow w$ that

$$
\limsup _{n \rightarrow \infty}\left\langle v-y_{n}, g\right\rangle=\langle v-w, g\rangle \geq 0
$$

It follows from the maximal monotonicity of $B+M$ that $\theta \in(M+B)(w)$, that is, $w \in I(B, M)$. Therefore, $w \in \Omega$. It follows that

$$
\limsup _{n \rightarrow \infty}\left\langle(\gamma f-A) q, S y_{n}-q\right\rangle=\lim _{i \rightarrow \infty}\left\langle(\gamma f-A) q, S y_{n_{i}}-q\right\rangle=\langle(\gamma f-A) q, w-q\rangle \leq 0
$$

Step 6. We prove that $x_{n} \rightarrow q$. By using (3.2) and together with Schwarz inequality, we have that

$$
\begin{aligned}
\left\|x_{n+1}-q\right\|^{2}= & \left\|P_{C}\left(\alpha_{n} \gamma f\left(x_{n}\right)+\left(I-\alpha_{n} A\right) S y_{n}\right)-P_{C}(q)\right\|^{2} \\
\leq & \left\|\alpha_{n}\left(\gamma f\left(x_{n}\right)-A q\right)+\left(I-\alpha_{n} A\right)\left(S y_{n}-q\right)\right\|^{2} \\
\leq & \left(I-\alpha_{n} A\right)^{2}\left\|\left(S y_{n}-q\right)\right\|^{2}+\alpha_{n}^{2}\left\|\gamma f\left(x_{n}\right)-A q\right\|^{2} \\
& +2 \alpha_{n}\left\langle\left(I-\alpha_{n} A\right)\left(S y_{n}-q\right), \gamma f\left(x_{n}\right)-A q\right\rangle \\
\leq & \left(1-\alpha_{n} \bar{\gamma}\right)^{2}\left\|y_{n}-q\right\|^{2}+\alpha_{n}^{2}\left\|\gamma f\left(x_{n}\right)-A q\right\|^{2} \\
& +2 \alpha_{n}\left\langle S y_{n}-q, \gamma f\left(x_{n}\right)-A q\right\rangle-2 \alpha_{n}^{2}\left\langle A\left(S y_{n}-q\right), \gamma f\left(x_{n}\right)-A q\right\rangle \\
\leq & \left(1-\alpha_{n} \bar{\gamma}\right)^{2}\left\|x_{n}-q\right\|^{2}+\alpha_{n}^{2}\left\|\gamma f\left(x_{n}\right)-A q\right\|^{2}+2 \alpha_{n}\left\langle S y_{n}-q, \gamma f\left(x_{n}\right)-\gamma f(q)\right\rangle \\
& +2 \alpha_{n}\left\langle S y_{n}-q, \gamma f(q)-A q\right\rangle-2 \alpha_{n}^{2}\left\langle A\left(S y_{n}-q\right), \gamma f\left(x_{n}\right)-A q\right\rangle \\
\leq & \left(1-\alpha_{n} \bar{\gamma}\right)^{2}\left\|x_{n}-q\right\|^{2}+\alpha_{n}^{2}\left\|\gamma f\left(x_{n}\right)-A q\right\|^{2}+2 \alpha_{n}\left\|S y_{n}-q\right\|\left\|\gamma f\left(x_{n}\right)-\gamma f(q)\right\| \\
& +2 \alpha_{n}\left\langle S y_{n}-q, \gamma f(q)-A q\right\rangle-2 \alpha_{n}^{2}\left\langle A\left(S y_{n}-q\right), \gamma f\left(x_{n}\right)-A q\right\rangle \\
\leq & \left(1-\alpha_{n} \bar{\gamma}\right)^{2}\left\|x_{n}-q\right\|^{2}+\alpha_{n}^{2}\left\|\gamma f\left(x_{n}\right)-A q\right\|^{2}+2 \gamma \alpha \alpha_{n}\left\|y_{n}-q\right\|\left\|x_{n}-q\right\| \\
& +2 \alpha_{n}\left\langle S y_{n}-q, \gamma f(q)-A q\right\rangle-2 \alpha_{n}^{2}\left\langle A\left(S y_{n}-q\right), \gamma f\left(x_{n}\right)-A q\right\rangle \\
\leq & \left(1-\alpha_{n} \bar{\gamma}\right)^{2}\left\|x_{n}-q\right\|^{2}+\alpha_{n}^{2}\left\|\gamma f\left(x_{n}\right)-A q\right\|^{2}+2 \gamma \alpha \alpha_{n}\left\|x_{n}-q\right\|^{2} \\
& +2 \alpha_{n}\left\langle S y_{n}-q, \gamma f(q)-A q\right\rangle-2 \alpha_{n}^{2}\left\langle A\left(S y_{n}-q\right), \gamma f\left(x_{n}\right)-A q\right\rangle
\end{aligned}
$$




$$
\begin{aligned}
& \leq\left(\left(1-\alpha_{n} \bar{\gamma}\right)^{2}+2 \gamma \alpha \alpha_{n}\right)\left\|x_{n}-q\right\|^{2} \\
& +\alpha_{n}\left\{\alpha_{n}\left\|\gamma f\left(x_{n}\right)-A q\right\|^{2}+2\left\langle S y_{n}-q, \gamma f(q)-A q\right\rangle\right. \\
& \left.\quad-2 \alpha_{n}\left\|A\left(S y_{n}-q\right)\right\|\left\|\gamma f\left(x_{n}\right)-A q\right\|\right\} \\
& =\left(1-2(\bar{\gamma}-\gamma \alpha) \alpha_{n}\right)\left\|x_{n}-q\right\|^{2} \\
& +\alpha_{n}\left\{\alpha_{n}\left\|\gamma f\left(x_{n}\right)-A q\right\|^{2}+2\left\langle S y_{n}-q, \gamma f(q)-A q\right\rangle\right. \\
& \left.\quad-2 \alpha_{n}\left\|A\left(S y_{n}-q\right)\right\|\left\|\gamma f\left(x_{n}\right)-A q\right\|+\alpha_{n} \bar{\gamma}^{2}\left\|x_{n}-q\right\|^{2}\right\} .
\end{aligned}
$$

Since $\left\{x_{n}\right\}$ is bounded, where $\eta \geq\left\|\gamma f\left(x_{n}\right)-A q\right\|^{2}-2\left\|A\left(S y_{n}-q\right)\right\|\left\|\gamma f\left(x_{n}\right)-A q\right\|+$ $\bar{r}^{2}\left\|x_{n}-q\right\|^{2}$ for all $n \geq 0$, it follows that

$$
\left\|x_{n+1}-q\right\|^{2} \leq\left(1-2(\bar{\gamma}-\gamma \alpha) \alpha_{n}\right)\left\|x_{n}-q\right\|^{2}+\alpha_{n} \varsigma_{n}
$$

where $\varsigma_{n}=2\left\langle S y_{n}-q, \gamma f(q)-A q\right\rangle+\eta \alpha_{n}$. By $\limsup _{n \rightarrow \infty}\left\langle(\gamma f-A) q, S y_{n}-q\right\rangle \leq 0$, we get $\lim \sup _{n \rightarrow \infty} S_{n} \leq 0$. Applying Lemma 2.5, we can conclude that $x_{n} \rightarrow q$. This completes the proof.

Corollary 3.2. Let $H$ be a real Hilbert space and $C$ a closed convex subset of $H$. Let $B, \Psi: C \rightarrow H$ be $\beta, \sigma$-inverse-strongly monotone mappings and $\varphi: C \rightarrow \mathcal{R}$ a convex and lower semicontinuous function. Let $f: C \rightarrow C$ be a contraction with coefficient $\alpha(0<\alpha<1), M: H \rightarrow 2^{H}$ a maximal monotone mapping, and $S$ a nonexpansive mapping of $C$ into itself such that

$$
\Omega:=F(S) \cap \operatorname{GMEP}(F, \varphi, \Psi) \cap I(B, M) \neq \emptyset .
$$

Suppose that $\left\{x_{n}\right\}$ is a sequence generated by the following algorithm for $x_{0}, u_{n} \in C$ arbitrarily:

$$
\begin{gathered}
F\left(u_{n}, y\right)+\varphi(y)-\varphi\left(u_{n}\right)+\left\langle\Psi x_{n}, y-u_{n}\right\rangle+\frac{1}{r_{n}}\left\langle y-u_{n}, u_{n}-x_{n}\right\rangle \geq 0, \quad \forall y \in C, \\
x_{n+1}=P_{C}\left[\alpha_{n} f\left(x_{n}\right)+\left(1-\alpha_{n}\right) S J_{M, \lambda}\left(u_{n}-\lambda B u_{n}\right)\right]
\end{gathered}
$$

for all $n=0,1,2, \ldots$, by (C1)-(C3) in Theorem 3.1.

Then $\left\{x_{n}\right\}$ converges strongly to $q \in \Omega$, where $q=P_{\Omega}(f+I)(q)$ which solves the following variational inequality:

$$
\langle(f-I) q, p-q\rangle \leq 0, \quad \forall p \in \Omega
$$

Proof. Putting $A \equiv I$ and $\gamma \equiv 1$ in Theorem 3.1, we can obtain the desired conclusion immediately. 
Corollary 3.3. Let $H$ be a real Hilbert space and $C$ a closed convex subset of $H$. Let $B, \Psi: C \rightarrow H$ be $\beta, \sigma$-inverse-strongly monotone mappings, $\varphi: C \rightarrow R$ a convex and lower semicontinuous function, and $M: H \rightarrow 2^{H}$ a maximal monotone mapping. Let $S$ be a nonexpansive mapping of $C$ into itself such that

$$
\Omega:=F(S) \cap \operatorname{GMEP}(F, \varphi, \Psi) \cap I(B, M) \neq \emptyset .
$$

Suppose that $\left\{x_{n}\right\}$ is a sequence generated by the following algorithm for $x_{0}, u \in C$ and $u_{n} \in C$ :

$$
\begin{gathered}
F\left(u_{n}, y\right)+\varphi(y)-\varphi\left(u_{n}\right)+\left\langle\Psi x_{n}, y-u_{n}\right\rangle+\frac{1}{r_{n}}\left\langle y-u_{n}, u_{n}-x_{n}\right\rangle \geq 0, \quad \forall y \in C \\
x_{n+1}=P_{C}\left[\alpha_{n} u+\left(1-\alpha_{n}\right) S J_{M, \lambda}\left(u_{n}-\lambda B u_{n}\right)\right]
\end{gathered}
$$

for all $n=0,1,2, \ldots$, by (C1)-(C3) in Theorem 3.1.

Then $\left\{x_{n}\right\}$ converges strongly to $q \in \Omega$, where $q=P_{\Omega}(q)$ which solves the following variational inequality:

$$
\langle u-q, p-q\rangle \leq 0, \quad \forall p \in \Omega .
$$

Proof. Putting $f(x) \equiv u$, for all $x \in C$, in Corollary 3.2, we can obtain the desired conclusion immediately.

Corollary 3.4. Let $H$ be a real Hilbert space, $C$ a closed convex subset of $H, B: C \rightarrow H$ be $\beta$ inverse-strongly monotone mappings, and $A$ a strongly positive linear bounded operator of $H$ into itself with coefficient $\bar{\gamma}>0$. Assume that $0<\gamma<\bar{\gamma} / \alpha$. Let $f: C \rightarrow C$ be a contraction with coefficient $\alpha(0<\alpha<1)$ and $S$ a nonexpansive mapping of $C$ into itself such that

$$
\Omega:=F(S) \cap V I(C, B) \neq \emptyset .
$$

Suppose that $\left\{x_{n}\right\}$ is a sequence generated by the following algorithm for $x_{0} \in C$ arbitrarily:

$$
x_{n+1}=P_{C}\left[\alpha_{n} \gamma f\left(x_{n}\right)+\left(I-\alpha_{n} A\right) S P_{C}\left(x_{n}-\lambda B x_{n}\right)\right]
$$

for all $n=0,1,2, \ldots$, by $(C 1)-(C 3)$ in Theorem 3.1.

Then $\left\{x_{n}\right\}$ converges strongly to $q \in \Omega$, where $q=P_{\Omega}(\gamma f+I-A)(q)$ which solves the following variational inequality:

$$
\langle(\gamma f-A) q, p-q\rangle \leq 0, \quad \forall p \in \Omega .
$$

Proof. Taking $F \equiv 0, \Psi \equiv 0, \varphi \equiv 0, u_{n}=x_{n}$, and $J_{M, \lambda}=P_{C}$ in Theorem 3.1, we can obtain the desired conclusion immediately.

Remark 3.5. In Corollary 3.4 we generalize and improve the result of Klin-eam and Suantai [24]. 


\section{Applications}

In this section, we apply the iterative scheme (1.25) for finding a common fixed point of nonexpansive mapping and strictly pseudocontractive mapping and also apply Theorem 3.1 for finding a common fixed point of nonexpansive mappings and inverse-strongly monotone mappings.

Definition 4.1. A mapping $T: C \rightarrow C$ is called strictly pseudocontraction if there exists a constant $0 \leq \kappa<1$ such that

$$
\|T x-T y\|^{2} \leq\|x-y\|^{2}+\kappa\|(I-T) x-(I-T) y\|^{2}, \quad \forall x, y \in C .
$$

If $\kappa=0$, then $S$ is nonexpansive. In this case, we say that $T: C \rightarrow C$ is a $\kappa$-strictly pseudocontraction. Putting $B=I-T$. Then, we have that

$$
\|(I-B) x-(I-B) y\|^{2} \leq\|x-y\|^{2}+\kappa\|B x-B y\|^{2}, \quad \forall x, y \in C .
$$

Observe that

$$
\|(I-B) x-(I-B) y\|^{2}=\|x-y\|^{2}+\|B x-B y\|^{2}-2\langle x-y, B x-B y\rangle, \quad \forall x, y \in C .
$$

Hence, we obtain

$$
\langle x-y, B x-B y\rangle \geq \frac{1-\kappa}{2}\|B x-B y\|^{2}, \quad \forall x, y \in C .
$$

Then, $B$ is $((1-\kappa) / 2)$-inverse-strongly monotone mapping.

Using Theorem 3.1, we first prove a strong convergence theorem for finding a common fixed point of a nonexpansive mapping and a strict pseudocontraction.

Theorem 4.2. Let $H$ be a real Hilbert space, $C$ a closed convex subset of $H, B, \Psi: C \rightarrow H$ be $\beta$, $\sigma$-inverse-strongly monotone mappings, $\varphi: C \rightarrow \mathcal{R}$ a convex and lower semicontinuous function, $f: C \rightarrow C$ a contraction with coefficient $\alpha(0<\alpha<1)$, and $A$ a strongly positive linear bounded operator of $H$ into itself with coefficient $\bar{\gamma}>0$. Assume that $0<\gamma<\bar{\gamma} / \alpha$. Let $S$ be a nonexpansive mapping of $C$ into itself, and let $T$ be a $\kappa$-strictly pseudocontraction of $C$ into itself such that

$$
\Omega:=F(S) \cap F(T) \cap \operatorname{GMEP}(F, \varphi, \Psi) \neq \emptyset .
$$

Suppose that $\left\{x_{n}\right\}$ is a sequence generated by the following algorithm for $x_{0}, u_{n} \in C$ arbitrarily:

$$
\begin{gathered}
F\left(u_{n}, y\right)+\varphi(y)-\varphi\left(u_{n}\right)+\left\langle\Psi x_{n}, y-u_{n}\right\rangle+\frac{1}{r_{n}}\left\langle y-u_{n}, u_{n}-x_{n}\right\rangle \geq 0, \quad \forall y \in C, \\
x_{n+1}=P_{C}\left[\alpha_{n} \gamma f\left(x_{n}\right)+\left(I-\alpha_{n} A\right) S\left((1-\lambda) u_{n}+\lambda T u_{n}\right)\right]
\end{gathered}
$$

for all $n=0,1,2, \ldots$, by $(C 1)-(C 3)$ in Theorem 3.1. 
Then $\left\{x_{n}\right\}$ converges strongly to $q \in \Omega$, where $q=P_{\Omega}(\gamma f+I-A)(q)$ which solves the following variational inequality:

$$
\langle(\gamma f-A) q, p-q\rangle \leq 0, \quad \forall p \in \Omega
$$

which is the optimality condition for the minimization problem

$$
\min _{q \in \Omega} \frac{1}{2}\langle A q, q\rangle-h(q)
$$

where $h$ is a potential function for $\gamma f\left(\right.$ i.e., $h^{\prime}(q)=\gamma f(q)$ for $\left.q \in H\right)$.

Proof. Put $B \equiv I-T$, then $B$ is $((1-\kappa) / 2)$-inverse-strongly monotone, $F(T)=I(B, M)$, and $J_{M, \lambda}\left(x_{n}-\lambda B x_{n}\right)=(1-\lambda) x_{n}+\lambda T x_{n}$. So by Theorem 3.1, we obtain the desired result.

Corollary 4.3. Let $H$ be a real Hilbert space, $C$ a closed convex subset of $H, B, \Psi: C \rightarrow H$ be $\beta, \sigma-$ inverse-strongly monotone mappings, and $\varphi: C \rightarrow R$ a convex and lower semicontinuous function. Let $f: C \rightarrow C$ be a contraction with coefficient $\alpha(0<\alpha<1)$ and $S$ a nonexpansive mapping of $C$ into itself, and let $T$ be a $\kappa$-strictly pseudocontraction of $C$ into itself such that

$$
\Omega:=F(S) \cap F(T) \cap \operatorname{GMEP}(F, \varphi, \Psi) \neq \emptyset .
$$

Suppose that $\left\{x_{n}\right\}$ is a sequence generated by the following algorithm for $x_{0} \in C$ arbitrarily:

$$
\begin{gathered}
F\left(u_{n}, y\right)+\varphi(y)-\varphi\left(u_{n}\right)+\left\langle\Psi x_{n}, y-u_{n}\right\rangle+\frac{1}{r_{n}}\left\langle y-u_{n}, u_{n}-x_{n}\right\rangle \geq 0, \quad \forall y \in C \\
x_{n+1}=P_{C}\left[\alpha_{n} f\left(x_{n}\right)+\left(I-\alpha_{n}\right) S\left((1-\lambda) u_{n}+\lambda T u_{n}\right)\right]
\end{gathered}
$$

for all $n=0,1,2, \ldots$, by (C1)-(C3) in Theorem 3.1.

Then $\left\{x_{n}\right\}$ converges strongly to $q \in \Omega$, where $q=P_{\Omega}(f+I)(q)$ which solves the following variational inequality:

$$
\langle(f-I) q, p-q\rangle \leq 0, \quad \forall p \in \Omega
$$

which is the optimality condition for the minimization problem

$$
\min _{q \in \Omega} \frac{1}{2}\langle A q, q\rangle-h(q)
$$

where $h$ is a potential function for $\gamma f$ (i.e., $h^{\prime}(q)=\gamma f(q)$ for $\left.q \in H\right)$.

Proof. Putting $A \equiv I$ and $\gamma \equiv 1$ in Theorem 4.2, we obtain the desired result. 


\section{Acknowledgments}

The authors would like to thank the National Research University Project of Thailand's Office of the Higher Education Commission for financial support under the project NRU-CSEC no. 54000267. Furthermore, they also would like to thank the Faculty of Science (KMUTT) and the National Research Council of Thailand. Finally, the authors would like to thank Professor Vittorio Colao and the referees for reading this paper carefully, providing valuable suggestions and comments, and pointing out a major error in the original version of this paper.

\section{References}

[1] F. E. Browder, "Existence and approximation of solutions of nonlinear variational inequalities," Proceedings of the National Academy of Sciences of the United States of America, vol. 56, pp. 1080-1086, 1966.

[2] E. Blum and W. Oettli, "From optimization and variational inequalities to equilibrium problems," The Mathematics Student, vol. 63, no. 1-4, pp. 123-145, 1994.

[3] P. L. Combettes and S. A. Hirstoaga, "Equilibrium programming in Hilbert spaces," Journal of Nonlinear and Convex Analysis, vol. 6, no. 1, pp. 117-136, 2005.

[4] S. D. Flåm and A. S. Antipin, "Equilibrium programming using proximal-like algorithms," Mathematical Programming, vol. 78, no. 1, pp. 29-41, 1997.

[5] S. Takahashi and W. Takahashi, "Viscosity approximation methods for equilibrium problems and fixed point problems in Hilbert spaces," Journal of Mathematical Analysis and Applications, vol. 331, no. 1 , pp. 506-515, 2007.

[6] P. Hartman and G. Stampacchia, “On some non-linear elliptic differential-functional equations," Acta Mathematica, vol. 115, pp. 271-310, 1966.

[7] J.-C. Yao and O. Chadli, "Pseudomonotone complementarity problems and variational inequalities," in Handbook of Generalized Convexity and Generalized Monotonicity, vol. 76 of Nonconvex Optim. Appl., pp. 501-558, Springer, New York, NY, USA, 2005.

[8] G. Marino and H.-K. Xu, "A general iterative method for nonexpansive mappings in Hilbert spaces," Journal of Mathematical Analysis and Applications, vol. 318, no. 1, pp. 43-52, 2006.

[9] W. A. Kirk, "A fixed point theorem for mappings which do not increase distances," The American Mathematical Monthly, vol. 72, pp. 1004-1006, 1965.

[10] Y. Hao, "Some results of variational inclusion problems and fixed point problems with applications," Applied Mathematics and Mechanics. English Edition, vol. 30, no. 12, pp. 1589-1596, 2009.

[11] M. Liu, S. S. Chang, and P. Zuo, "An algorithm for finding a common solution for a system of mixed equilibrium problem, quasivariational inclusion problem, and fixed point problem of nonexpansive semigroup," Journal of Inequalities and Applications, vol. 2010, Article ID 895907, 23 pages, 2010.

[12] J. F. Tan and S. S. Chang, "Iterative algorithms for finding common solutions to variational inclusion equilibrium and fixed point problems," Fixed Point Theory and Applications, vol. 2011, Article ID 915629, 17 pages, 2011.

[13] S.-S. Zhang, J. H. W. Lee, and C. K. Chan, "Algorithms of common solutions to quasi variational inclusion and fixed point problems," Applied Mathematics and Mechanics. English Edition, vol. 29, no. 5, pp. 571-581, 2008.

[14] R. T. Rockafellar, "On the maximality of sums of nonlinear monotone operators," Transactions of the American Mathematical Society, vol. 149, pp. 75-88, 1970.

[15] B. Lemaire, "Which fixed point does the iteration method select?" in Recent Advances in Optimization (Trier, 1996), vol. 452 of Lecture Notes in Econom. and Math. Systems, pp. 154-167, Springer, Berlin, Germany, 1997.

[16] A. Moudafi, "Viscosity approximation methods for fixed-points problems," Journal of Mathematical Analysis and Applications, vol. 241, no. 1, pp. 46-55, 2000.

[17] H. Iiduka and W. Takahashi, "Strong convergence theorems for nonexpansive mappings and inversestrongly monotone mappings," Nonlinear Analysis: Theory, Methods E Applications, vol. 61, no. 3, pp. 341-350, 2005. 
[18] Y. Su, M. Shang, and X. Qin, "An iterative method of solution for equilibrium and optimization problems," Nonlinear Analysis: Theory, Methods \& Applications, vol. 69, no. 8, pp. 2709-2719, 2008.

[19] H. Brézis, "Opérateur maximaux monotones," in Mathematics Studies, vol. 5, North-Holland, Amsterdam, The Netherlands, 1973.

[20] Z. Opial, "Weak convergence of the sequence of successive approximations for nonexpansive mappings," Bulletin of the American Mathematical Society, vol. 73, pp. 591-597, 1967.

[21] H.-K. Xu, "Iterative algorithms for nonlinear operators," Journal of the London Mathematical Society. Second Series, vol. 66, no. 1, pp. 240-256, 2002.

[22] F. E. Browder, "Nonlinear operators and nonlinear equations of evolution in Banach spaces," in Nonlinear Functional Analysis, pp. 1-308, Amer. Math. Soc., Providence, RI, USA, 1976.

[23] J.-W. Peng, Y.-C. Liou, and J.-C. Yao, "An iterative algorithm combining viscosity method with parallel method for a generalized equilibrium problem and strict pseudocontractions," Fixed Point Theory and Applications, vol. 2009, Article ID 794178, 21 pages, 2009.

[24] C. Klin-eam and S. Suantai, "A new approximation method for solving variational inequalities and fixed points of nonexpansive mappings," Journal of Inequalities and Applications, vol. 2009, Article ID 520301, 16 pages, 2009. 


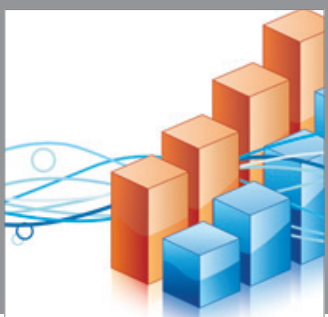

Advances in

Operations Research

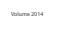

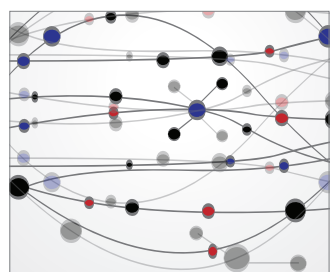

\section{The Scientific} World Journal
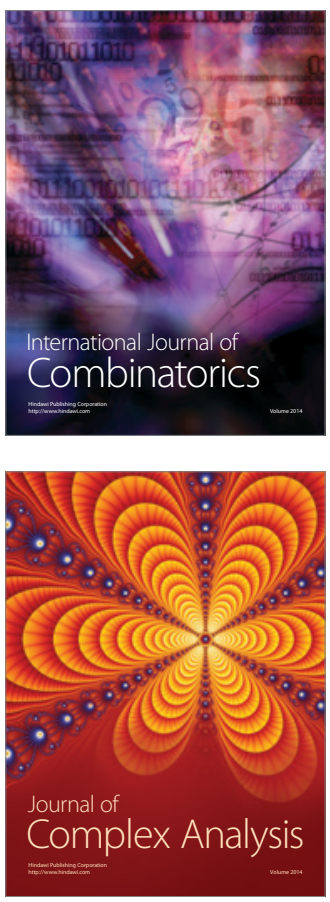

International Journal of

Mathematics and

Mathematical

Sciences
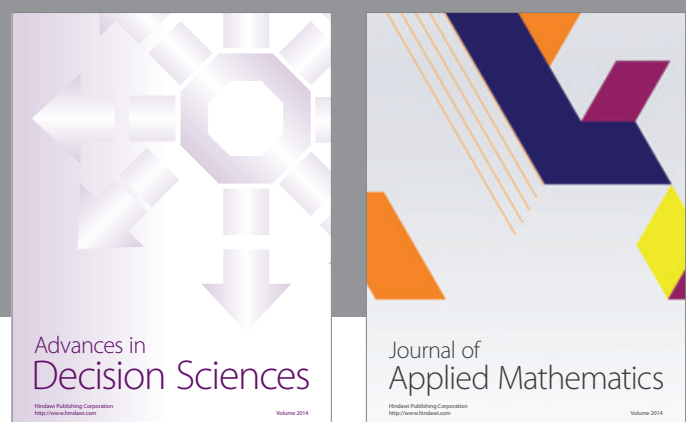

Journal of

Applied Mathematics
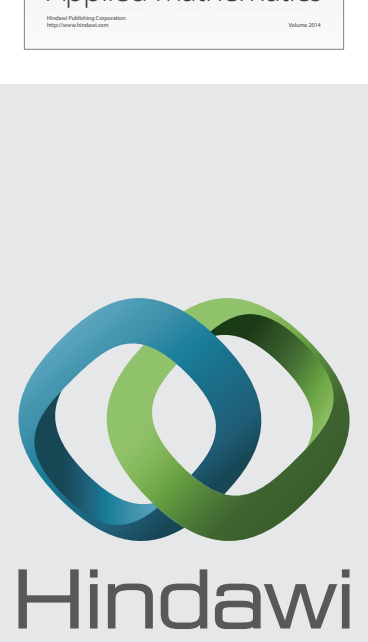

Submit your manuscripts at http://www.hindawi.com
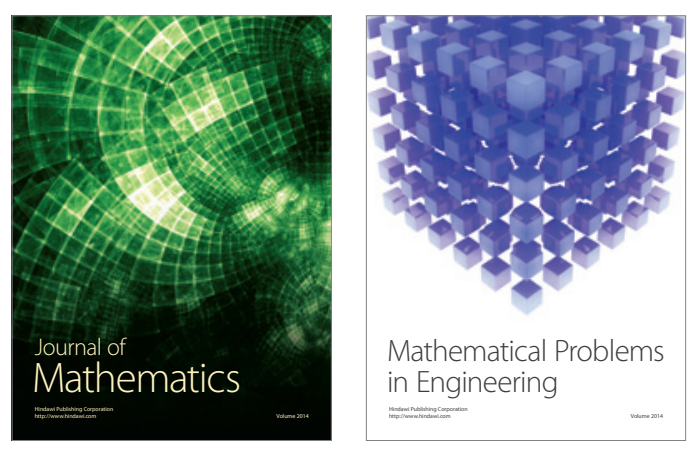

Mathematical Problems in Engineering
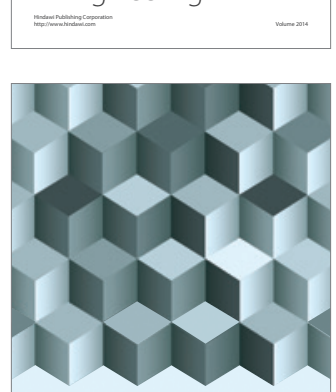

Journal of

Function Spaces
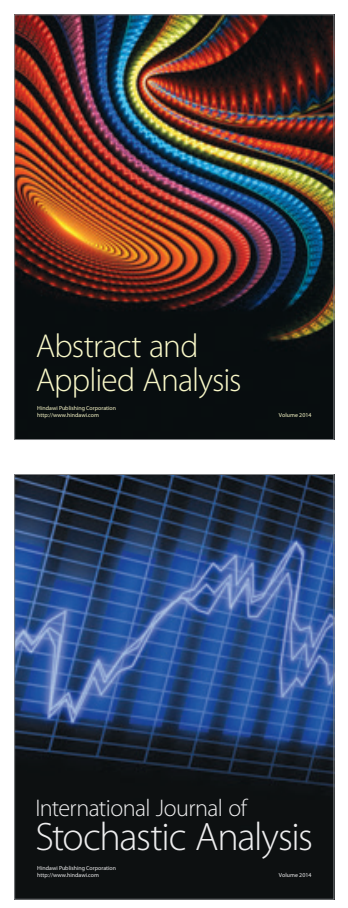

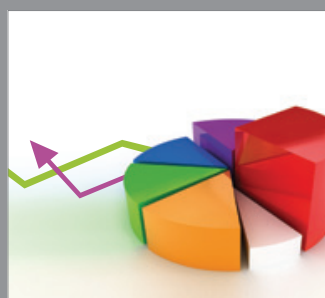

ournal of

Probability and Statistics

Promensencen
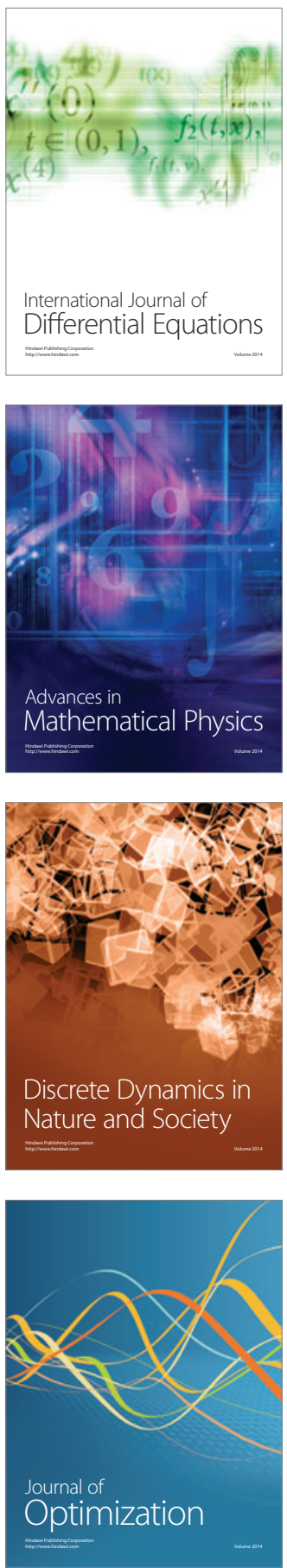